\title{
MAGNETIC Ap STARS: EVOLUTIONARY STATUS AND ABUNDANCE ANOMALIES
}

\author{
T. A. RYABCHIKOVA \\ The Astronomical Council of \\ the Academy of Sciences of the USSR \\ Pyatnitskaya str. 48, Moscow \\ 109017, U.S.S.R.
}

\begin{abstract}
Some general properties and evolutionary status of magnetic Ap stars are briefly considered. Chemical abundance anomalies are discussed from the point of view.of current theories presumably the radiative diffusion theory in the presence of magnetic field.
\end{abstract}

\section{INTRODUCTION}

The class of upper main-sequence magnetic Ap stars consists of several groups of stars showing unusual strengths of spectral lines of certain chemical elements in their spectra and having usually measurable surface magnetic fields of $10^{2}-10^{4} G s$. Almost all magnetic Ap stars are light, spectral and magnetic variables with the same period which is the star rotation period. All variations in magnetic Ap stars can be explained by the oblique rotator model (see review by Wolff, 1983, and references therein) with the inhomogeneous distribution of chemical elements on the stellar surface (see review by Khokhlova, 1983). A few of the coolest magnetic Ap stars are also pulsating stars with periods of 4-16 minutes. The model which best explains certain characteristic features of Ap star pulsations is the oblique pulsator model (see review by Kurtz, 1982, and references therein).

According to the Jaschek and Jaschek (1958) and Osawa (1965) classifications, magnetic Ap stars can be sorted into five main groups: the $\lambda 4200-\mathrm{Si}, \mathrm{Si}, \mathrm{Si}-\mathrm{Cr}-\mathrm{Eu}, \mathrm{Sr}-\mathrm{Cr}-\mathrm{Eu}$ and $\mathrm{Sr}$ groups. This sequence correlates statistically with their colours and as a result with their effective temperature. The bright star $\alpha^{2} C V n$ is a prototype of magnetic Ap stars.

\section{SOME GENERAL CHARACTERISTICS AND EVOLUTIONARY STATUS OF Ap STARS}

\subsection{Temperatures}

On the HR diagram magnetic Ap stars occupy a region of effective temperatures from 7500 $\mathrm{K}$ up to $15000 \mathrm{~K}$. At both temperature limits the groups of magnetic stars overlap with the groups of nonmagnetic peculiar stars: $\mathrm{Hg}$ - Mn stars lie in the hot temperature end while Am stars lie in the cool one. Ultraviolet observations of Ap stars reveal an ultravio- 
let flux deficiency in comparison with the normal stars of the same UBV colours which is presumably caused by enhanced line and continuum opacity in the region of $\lambda<3000 \mathrm{~A}$ (Leckrone et al. 1974). Therefore the effective temperatures obtained from the total luminosity are lower than those obtained from UBV calibrations for normal stars. New model atmosphere calculations by Muthsam (1979) take into account the enhanced line blanketing due to abundance anomalies of Ap stars and show that an Ap star atmosphere corresponds to that of normal star with a higher effective temperature. Recently several new temperature calibrations were proposed by Lanz (1985), Mégessier (1988) and Stẹpień and Dominiczak (1989). All these calibrations agree rather well and ensure the basis for accurate temperature determinations of Ap stars.

\subsection{Rotation}

It is a well-known fact that almost all Ap stars are slow rotators (see review by Wolff, 1983). Slow rotation together with magnetic field make the stellar atmosphere stable enough to allow chemical separation. In their investigation of stellar rotational velocities Ramella et al. (1989) show that all A0 stars of V luminosity class with $V \sin i<15 \mathrm{~km} / \mathrm{s}$ are chemically anomalous. The question is when Ap stars begin to rotate slowly? Wolff (1981) determined an overall decrease of rotational velocity with decreasing of mass and consequently with increasing of age. On the other hand North (1984) and Klochkova and Kopylov (1986) found that rotational velocity of Ap stars does not depend on stellar age and that an Ap star should loose its angular momentum before reaching the main sequence. In this connection we would like to refer to a recent paper by Ponomarev et al. (1989). While trying to interpret the maser recombination lines in MWC 349 (an emission line radio star) they had to assume that the star and its surrounding disc were formed from a premordial cloud with an "anomalously" small angular momentum. Is it really an anomaly or just a normal manifestation of the distribution of protostellar clouds upon their specific angular momentum? The statistical investigation can show whether there is any connection with the peculiar stars.

\subsection{Evolutionary status}

Two recent investigations of Ap stars radii (Stẹpień, 1989) and surface gravities (North and Kroll, 1989) show that all Ap stars lie within the main sequence strip and there are no stars with $\log g<3.0$. Loden and Sundman (1989) compared luminosities of Ap stars that were thought to be giants with those of normal main-sequence stars and "normal" Ap stars and found no differences between them. There are some indications of a slightly evolved stage of two cool pulsating Ap stars HD 24712 and HD 203932 obtained from the interpretation of their observed frequency patterns (Kurtz et al., 1989; Kurtz, 1988), but the question is not clear up to now. The most reliable data of stellar masses and radii can be obtained from eclipsing binaries. Ten eclipsing binaries with Ap primaries are known. Lebedev (1989) presented effective temperatures, masses, radii and luminosities for eight of them. All these stars fall within the main sequence strip. The two other eclipsing binaries are $\omega U M a$ (Hric, 1988) and TV Nor (Renson, 1989). Abundance analysis of such systems in the phases of light minima can give important results concerning the occurrance of peculiar chemical composition in the members of binary systems. 
Another question of Ap star evolution is when the star gets its abundance anomalies. Klochkova and Kopylov (1986) did not find any correlation of star peculiarity index with age (for $\mathrm{He}, \mathrm{Si}$ and Sr), while Mégessier (1986) presented an evidence of Si abundance decrease with age. North (1985) noted a constancy of equivalent widths of SiII doublet with age for stars with $M>3 M_{\odot}$, while for less massive stars there was a small tendency of decreasing $\mathrm{W}_{\lambda}$ with age. More uniform observations are necessary to clarify this question.

\section{ORIGIN OF ABUNDANCE ANOMALIES IN Ap STARS}

Trying to explain the observed abundance anomalies in Ap stars one must keep in mind the following observational facts:

1. Ap stars lie on or near main sequence and are not evolved objects.

2. Abundance anomalies are not global but rather surface characteristic of Ap stars.

3. Chemical elements are distributed nonuniformly on the stellar surface.

4. An evident connection exists between magnetic field and some of the chemical peculiarities.

5. Ap stars are usually slow rotators.

None of the suggested nuclear processes explains all these facts (see review by Wolff, 1983). Nonuclear processes seem to be more promising for the explanation of abundance anomalies in Ap stars. Three of them have been discussed in literature.

\subsection{Magnetic accretion}

Magnetic accretion of interstellar matter (Havnes and Conti, 1971) allows to explain in an approximate way some of the observed abundance anomalies especially if the interstellar matter is enriched by nuclear processed material from nearby supernovae. Recently Proffitt and Michaud (1989) discussed this problem and concluded that "only one A or B star in a few thousand is likely to show abundance anomalies caused by a nearby supernovae", a number which is obviously much less than observed frequency of Ap stars. Accretion onto the stellar surface occurs presumably near the magnetic poles and may explain surface abundance inhomogeneities. Another kind of magnetic accretion process has been recently proposed by Krishna Kumar et al. (1989). They considered the accretion of interplanetary dust by Ap and Am stars and made a conclusion that with the same comet bombarding rate, as observed in the solar system the proposed accretion process can predict the level of observed abundance ano malies in Ap and Am stars. Moreover they showed that in the case of dipolar magnetic field the distribution of observed anomalies must have a ringlike structure around magnetic poles without any anomalies occurred in place of poles themselves. As we shall see below such kind of chemical anomalies structure is observed in Ap stars.

\subsection{Light-induced drift}

Second nonuclear process has been investigated both theoretically and experimentally by Gel'mukhanov and Shalagin $(1979,1980)$. As a well-known radiative diffusion (Michaud, 1970 ) this mechanism belongs to the separation processes and can be shortly described as 
follows. Thermal velocities of particles in plasma lead to a Doppler broadening of spectral line. If we have some directed radiation asymmetric relative to the center of spectral line, atoms with appropriate velocity fall in resonance with the radiation frequency, become excited and change their effective scattering cross-section. In the presence of a buffer gas a difference in viscosity caused by the difference in cross-sections between excited atoms and those in the ground state leads to a stationary flow of considered atoms in a certain direction. So we have a case of chemical separation called light-induced drift (LID). Theoretical predictions and laboratory experiments show that the effective LID velocity is by some orders of magnitude higher than that caused by radiative pressure (Gel'mukhanov and Shalagin, 1979,1980). Atutov and Shalagin (1988) considered qualitatively an application of LID to stellar atmospheres and showed that for heavy elements at $T \sim 10000 \mathrm{~K}$ diffusion velocity must be of order of $1 \mathrm{~cm} / \mathrm{s}$. This mechanism may be most efficient in the producing of anomalous isotope ratios observed in peculiar stars but it requires detailed calculations for stellar atmospheres. Together with the convection LID can produce surface inhomogeneities as well as magnetic field generation (Atutov and Shalagin, 1988).

\subsection{Radiative diffusion}

The last nonuclear process considered here is a well-known radiative diffusion first proposed by Michaud (1970) and elaborated in a series of subsequent papers. An overall picture of predicted abundance anomalies under simplest assumptions neglecting the magnetic field was given by Michaud et al. (1976) and in review papers by Michaud (1980), Vauclair and Vauclair (1982), Alecian (1986, and references therein). Calculations of time dependent diffusion made by Alecian and Grappin (1984) showed that it is possible to have a stable solutions in optically thick layers, but before reaching it some transient unstable states may exist in Ap star atmosphere during stellar lifetime that lead to several phases of peculiarity for the same element in a given star. These transient states may explain the diversity of abundance anomalies observed in peculiar stars.

Michaud et al. (1981) have considered the problem of the influence of magnetic field on diffusion process. They have predicted the degree of anomalies as well as an element distribution over the surface of magnetic star. Some elements like that of iron peak should be concentrated in caps in the regions of vertical magnetic lines (near poles). Others such as $\mathrm{Si}$, rare-earth elements, can be supported only in the regions of horizontal magnetic lines or near magnetic equator giving the surface distribution in the form of rings. Horizontal diffusion quantitatively studied by Mégessier (1984) makes Si to migrate towards the magnetic poles where it sinks. This process takes about $10^{8}$ years. We can expect to observe age dependent silicon overabundance and silicon distribution over the stellar surface. Detailed diffusion calculations were also carried out for the following elements: B (Borsenberger et al. 1979); $\mathrm{Be}, \mathrm{Mg}, \mathrm{Ba}$ (Borsenberger et al. 1984); $\mathrm{Ca}$ and $\mathrm{Sr}$ (Borsenberger et al. 1981); Ga (Alecian and Artru, 1987) and Mn (Alecian and Michaud, 1981). Diffusion calculations show a temperature dependence of $\mathrm{Si}, \mathrm{Mn}$ and $\mathrm{Ga}$ overabundances.

In the next section we consider the measured abundances of magnetic Ap stars and compare them with the predictions of radiative diffusion theory as the most elaborated. 


\section{OBSERVED CHEMICAL ABUNDANCES OF MAGNETIC Ap STARS}

We shall consider general behaviour of mean abundances of magnetic Ap stars neglecting the surface spotty structure. In a few cases where the surface structure is known we shall also use it for comparison with the theoretical predictions.

Except Adelman (1973) abundance analysis of 21 cool Ap stars, improved later by Adelman and Cowley (1986), only scarce studies of chemical composition exist for magnetic Ap stars belonging to a different groups. We made a compilation of data obtained by model atmosphere analysis based on spectra with high enough quality containing the abundances for 11 stars of different effective temperatures and magnetic field strengths. This sample is not as homogeneous as the Adelman's one but nevertheless it can give us some ideas about behaviour of different elements in magnetic Ap stars. In all cases when the abundance survey of individual elements exists we prefer to use it as a primary source which may be supplemented by abundances of 11 stars. Here are references for these 11 stars: $\gamma E q u$ and HR 7575 (Allen and Cowley, 1977); $53 \mathrm{Cam}$ - mean abundances (Landstreet, 1988); 73 Dra (Sadakane, 1976); HD 2453, HD 8441 and HD 192913 (Ryabchikova and Ptitsyn, 1986); HR 465 (Aller, 1972); HD 25823 (Bolkal et al., 1987); HD 168733 (Little, 1974; Muthsam and Cowley, 1984); HD 215441 - mean values (Landstreet et al., 1989); cool Ap stars (Adelman and Cowley, 1986); Sun (Grevesse, 1984).

$\mathrm{He}, \mathrm{Li}, \mathrm{Be}$. He is underabundant in all Ap stars where it can be observed in agreement with the diffusion theory predictions. $\mathrm{Li}$ was found to be overabundant by 80 and 10 times in cool Sr-Cr-Eu stars HD 188041 and HD 201601 respectively while nothing was found at wavelength of LiI resonance doublet in the spectra of two other stars HD 15144 and HD 24712 , belonging to the same peculiar subgroup, as well as in the spectra of six stars of higher effective temperature (Faraggiana et al. 1986). No large overabundances of beryllium similar to those found in some $\mathrm{Hg}-\mathrm{Mn}$ stars have been found in these four cool $\mathrm{Sr}-\mathrm{Cr}-\mathrm{Eu}$ stars (Gerbaldi et al. 1986). Only in HD188041 Be may be 10 times the cosmic value. This result does not agree with diffusion theory predictions for stars of two solar mass (Vauclair et al. 1978) which suggest a significant Be overabundance while the observed lithium abundance is more consistent with diffusion.

$\underline{\mathbf{C}, \mathbf{N}, \mathbf{O}}$. CNO abundances in Ap stars were considered in detail in recent paper by Roby and Lambert (1990). They obtained the average values $[X / H]$ of $\mathrm{C}, \mathrm{N}$ and $\mathrm{O}$ for different groups of stars: $\mathrm{Hg}-\mathrm{Mn}$ stars $(+0.1,-0.7,-0.3)$, Si stars $(-0.2,-0.2,-0.5), \mathrm{Sr}-\mathrm{Cr}-$ Eu stars $(-0.7,-0.6,-1.3)$ and Am stars $(-0.2,-0.2,-0.4)$. Based on a larger sample of Ap stars Gerbaldi et al. (1989) went to the same conclusion about oxygen deficiency in their investigation of $O I$ infrared triplet. They stressed the large diversity of oxygen abundances in the intermediate temperature Ap stars of $\mathrm{Si}-\mathrm{Cr}-\mathrm{Eu}$ group. While there is some trend of $\mathrm{C}$ and $\mathrm{O}$ abundances with temperature, the latter is not the only parameter which defines this trend. At a given temperature both elements are usually more deficient in magnetic stars. Temperature behaviour of nitrogen in magnetic and nonmagnetic stars is quite different. The results for magnetic stars only roughly follow the simple diffusion predictions while a strong disagreement exists in the case of nitrogen in nonmagnetic stars. In both papers abundance analysis have been based mainly on spectral lines of neutral atoms. Using lines of $C I I$ and $N I I$ in visual spectral region Leushin et al. (this symposium) obtained a rather different result for five hot $\mathrm{Si}$ stars. Small overabundances of carbon are accompanied by 
relatively large nitrogen overabandances in these stars. This result is more close to what is predicted by the diffusion theory for a star with $T_{\text {eff }}=12500 \mathrm{~K}$ (Roby and Lambert, 1990) while the observed overabundances are slightly higher. $\alpha^{2} C V n$ is the only common object in two investigations. Roby and Lambert (1990) also noted an 1.0 dex discrepancy in abundance obtained from $C I$ and $C I I$ lines for hot magnetic stars. This question is waiting to be resolved.

Mg and $\mathrm{Al} . \mathrm{Mg}$ is more or less normal in magnetic Ap stars in good agreement with the diffusion theory predictions. $\mathrm{Al}$ is underabundant by about $1.0 \mathrm{dex}$ in $\mathrm{Sr}-\mathrm{Cr}-\mathrm{Eu}$ and $\mathrm{Si}$ stars except for the hot star HD 34452 (Sadakane et al. 1983) and intermediate temperature stars HR 465 and HD 192913, where it seems to be normal. Observed abundance of $\mathrm{Al}$ do not conforms with the suggestion of the diffusion theory.

Si. Silicon is the most studied element in Ap stars both theoretically and observationally. Some correlations of abundance with temperature, magnetic field intensity (Mégessier, 1986) and less evident - with age (see section 2.3 of this review) are in good agreement with the predictions of time dependent radiative diffusion theory in the presence of magnetic field (Mégessier, 1984). Silicon abundance distribution in the form of ring-like features in the regions of magnetic equator is obtained for $C U V i r$ (Goncharskij et al. 1983; Hatzes, 1988), $\gamma^{2}$ Ari (Hatzes et al., 1989), $\theta$ Aur (Hatzes, 1990). Using another SiII line Rice and Wehlau (1990) also obtained some ring-like distribution of Si in $\theta$ Aur but with the different axis of symmetry in comparison with the Hatzes distribution. In any case such a distribution roughly corresponds to what is expected from the selective silicon diffusion in the presence of dipolar magnetic field. But this diffusion scenario seems fail to explain silicon abundance cap near the strong magnetic pole where field lines are vertical to the surface of the evidently unevolved star HD 215441 with the strongest known surface magnetic field of $34 k G s$ (Landstreet et al. 1989). Another fact that is difficult to explain in the framework of current diffusion theories, is an existence of unevolved magnetic stars with silicon deficiency. The average underabundance of $\mathrm{Si}$ is so strong, that even in the silicon spots on the surface of $\epsilon U M a$ Totochava and Khokhlova (1990) determined a small silicon underabundance of -0.4 dex. Zverko et al. (1990) found silicon deficiency in the atmosphere of hotter Si star 68 Ori $=$ HR 2193 with $T_{\text {eff }}=11900 \mathrm{~K}$.

$\mathrm{Ca}$ and Sc. Calcium is more or less normal or slightly underabundant in Ap stars in agreement with the diffusion theory predictions without magnetic field. But it should be kept in mind a strong variability of Ca lines in some stars apparently related with the magnetic field variations (see, for example, Faraggiana (1973) study of $53 \mathrm{Cam}$ ). The observed underabundance of scandium is inconsistent but not dramatically with the radiative diffusion.

Iron peak elements. For $\mathrm{Mn}, \mathrm{Fe}, \mathrm{Co}$ and $\mathrm{Ni}$ which are produced in the same nuclear process we could not find any significant deviations of relative abundances from solar ones. Only for hotter star, HD 192913, there is probably a nickel overdeficiency. Iron abundance distribution in Ap stars was statistically studied by Cowley and Aikman (1980) who found it to be very close to those for normal stars with remarkably small dispersion of +0.5 dex. Iron abundance analysis using model atmosphere method confirms the same dispersion but gives higher mean value of $\log (\mathrm{Fe} / \mathrm{H})$ by the order of $+0.7 \mathrm{dex}$. Overabundances of $\mathrm{Mn}$, $\mathrm{Co}$ and $\mathrm{Ni}$ are small compared with the diffusion theory predictions.

According to Michaud et al. (1981) iron must be concentrated near the magnetic poles 
where field lines are vertical. It seems to be the case for 53 Cam (Landstreet, 1988) and for HD 215441 (Landstreet et al., 1989), while in $\alpha^{2} C V n$ (Khokhlova and Pavlova, 1984) iron is mainly concentrated in the equatorial regions. In all stars with known iron surface abundance distribution high horizontal gradients are not observed. But the picture is absolutely different in case of $\mathrm{Ti}$ and $\mathrm{Cr}$. Both elements are highly peculiar in magnetic Ap stars and show strongly inhomogeneous surface distribution in some cases. But if $\mathrm{Cr}$ overabundance does not depend on temperature and magnetic field strength, the behaviour of titanium is quite different. This is illustrated in Fig.1. All data are taken from the papers cited in the beginning of this Section. Inspite of poor sample of stars a clear dependence both upon temperature and magnetic field is seen. This relation is supported by other hot stars, HD 32633 with strong effective magnetic field, HD 24155, HD 122532 (Lanz and Landstreet, this symposium) and 108 Aqr (Mégessier et al. 1979). HD 32633 and 108 Aqr have an effective temperature about $12500 \mathrm{~K}$ and the titanium overabundance exceeds +3.0 dex at least on the part of their surface. In extremely peculiar star HD 215441 the abundances of $\mathrm{Ti}, \mathrm{Cr}$ and $\mathrm{Fe}$ are nearly equal in the region of strong magnetic pole! Diffusion theory predicts neither such a large titanium overabundance nor an existence of temperature dependance. Maximum of iron peak elements distribution seems to be shifted towards lighter even elements in hot magnetic stars. For magnetic Ap stars there are no cases of $(M n / F e)>1$ that is observed in several Hg-Mn stars (Allen and Cowley, 1977).

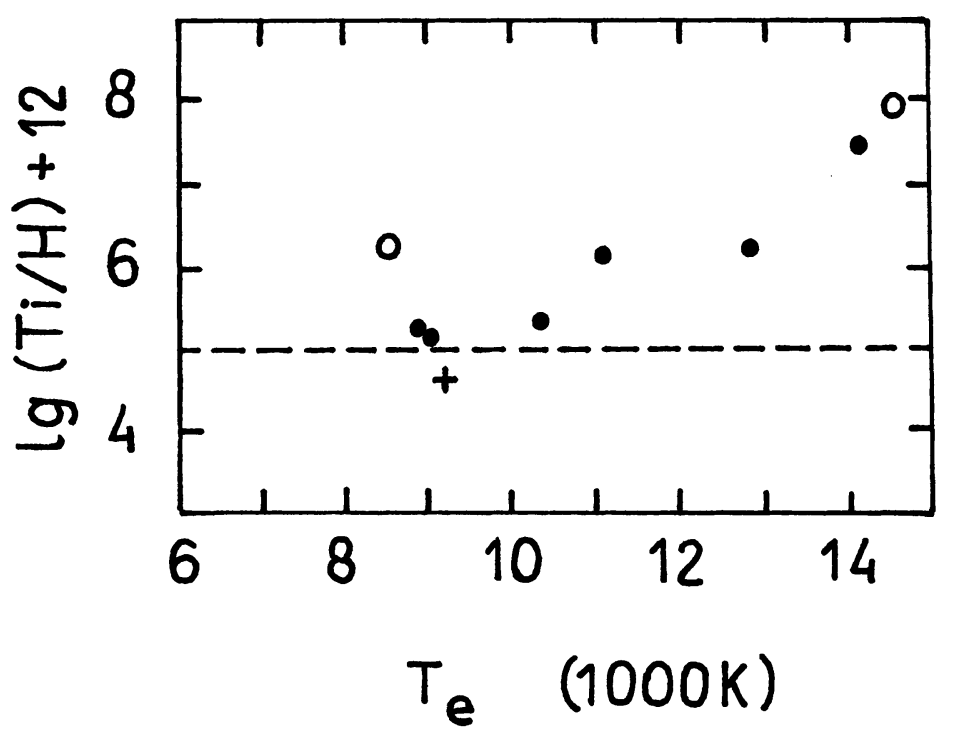

Figure 1. A plot of titanium abundances versus effective temperature for magnetic Ap stars. Dots represent stars with $H_{s}=1.5-3.5 k G s$, cross represents HD $8441\left(H_{s}=0.5\right)$, open circles represent $53 \mathrm{Cam}\left(H_{s}=15 k G s\right)$ and HD $215441\left(H_{s}=34 k G s\right)$. The solar $\mathrm{Ti}$ abundance is indicated by the dashed line. 
Ga. Gallium abundance study is mainly based on Takada-Hidai et al. (1986) paper. Using ultraviolet resonance $G a I I$ and $G a I I I$ lines they obtained a slightly less gallium overabundances in $\mathrm{Si}$ stars than in $\mathrm{Hg}-\mathrm{Mn}$ stars. This result is in good agreement with the predictions of gallium diffusion in the presence of magnetic field (Alecian and Artru, 1987). Moreover antiphase variation of $\mathrm{Ga}$ and $\mathrm{Si}$ lines intensity in HD 25823 also supports this theory (Artru and Freire-Ferrero, 1988). It should be noted that gallium abundance obtained from lines in the visual spectral region in this star is one order of magnitude higher than that obtained from lines in the ultraviolet spectral region (Lanz et al., this symposium).

Sr, Y, Zr and Ba. Strontium is typically 1.0-2.5 dex overabundant in magnetic Ap stars. Cowley and Aikman (1980) have noted a larger dispersion in yttrium abundance distribution. It is caused by tendency of $\mathrm{Y}$ and $\mathrm{Zr}$ abundances to correlate with temperature while no correlation is apparent for Sr. This was first noted by Allen (1977) for Hg-Mn stars. Yttrium and zirconium abundances for stars from our sample plotted on the Allen's Figs. 5 and 6 provide a remarkable consistency between magnetic and non magnetic stars. While there is no clear evidence of nonuclear abundance pattern for $\mathrm{Sr}-\mathrm{Y}-\mathrm{Zr}$, yttrium is often overabundant relative to its neighbors compared to the solar case. This behaviour of $\mathrm{Y}$ can not be explained in the framework of current diffusion theory.

Cowley (1976) has studied barium in normal, Am, and magnetic Ap stars and has concluded that there is no clear difference between $\mathrm{Ap}$ and normal groups. $\mathrm{Ba}$ is solar or slightly underabundant in cool Ap stars while towards the hotter ones $\left(T_{\text {eff }}>9000 \mathrm{~K}\right) \mathrm{Ba}$ overabundance is increased up to $1.5 \mathrm{dex}$. It is interesting to note the same behaviour of $\mathrm{Ba}$ abundance in normal stars. Am stars are more Ba overabundant than their Ap congeners.

Rare-earth elements. The problem of rare-earths or lanthanides in magnetic Ap stars has been investigated in series of papers by Cowley $(1976,1980,1983,1984)$. First by the method of Wavelength Coincidence Statistics and later (Magazzu and Cowley, 1986) by the model atmosphere abundance analysis a general similarity in lanthanides abundance pattern was established between cool Ap and Am stars. Moreover there is some statistical evidence that lanthanides abundance pattern of cool Ap stars is close to that of normal and peculiar late-type stars (Cowley, 1983). The situation on the hot side of magnetic Ap stars is quite different. Spectra of the most of hot Ap stars are replete with rareearth lines of first and second ions while there is no clear evidence of lanthanides in the spectra of $\mathrm{Hg}-\mathrm{Mn}$ stars. Lanthanide lines are also weak or absent in the spectra of hot Am stars Sirius and 68 Tau. Simple diffusion theory seems to fail in the case of rareearth elements. Cowley (1976) suggested that accretion theories were more promising for explanation of rare-earth elements in Ap and Am stars. Accretion theories also agree with the fact that practically in all stars maximum of rare-earth lines intensity coincides with one of the magnetic field extremum strongly suggesting the rare-earths concentration near the magnetic poles (Goncharskij et al., $1983\left(\alpha^{2} \mathrm{CVn}\right)$; Floquet, 1982 (52 Her and HD 25354), for example), while radiative diffusion in magnetic field predicts a rare-earth overabundance belt in the region of magnetic equator (Michaud et al., 1981).

Another problem for rare-earth elements is a high overabundance in hotter Ap stars obtained from analysis of singly ionized lines. Rough estimation of $\mathrm{Er}$ and $\mathrm{Sm}$ abundances using ErIII and SmIII lines in HR 465 (Cowley and Greenberg, 1987) leads to about two order lower values compared to that obtained from singly ionized lines. Comparison of 
ultraviolet spectra of HR 465 and normal stars reveals a similarity of $E u I I I$ resonance line intensities in these stars (Fuhrmann, 1989b), while overabundance of more than 3.0 dex results from analysis of visible EuII lines. Similar problems have been found by Lecrone (1976) in his analysis of ultraviolet spectrum of $\alpha^{2} C V n$. Rare-earth elements are mostly double ionized in the atmospheres of Ap stars. Cowley (1980) suggested that high rareearth overabundances in the atmospheres of some hotter stars where the observed single ionized lines do not represent the dominant ionization stage "may be due to a breakdown in the Saha equation or possibly to the existence of a cool upper photosphere caused by line blanketing in the hotter Ap stars". A lack of atomic data does not allow to use lines of double ionized lanthanides to examine this problem more accurately.

Heavy elements. Spectral lines PtII, AuII and $H g I I$ were identified in ultraviolet spectra of three Hg-Mn and two Ap stars HR 465 and $\alpha^{2} C V n$ (Fuhrmann, 1989a) although an identification of $A u I I$ is unreliable. The lines of platinum and bismuth were also identified in HR 465 by Cowley (1987) and BiII identification was supported by Fuhrmann. The presence of BiII lines has been established earlier only for one Hg-Mn star HR 7775 by Jacobs and Dworetsky (1982) and Guthrie (1984). After Fuhrman we can conclude that some similarity indeed exists between the hotter magnetic Ap and non magnetic $\mathrm{Hg}-\mathrm{Mn}$ stars. Another evidence of this similarity is HD 213918, a hot Si star with some characteristics of $\mathrm{Hg}-\mathrm{Mn}$ stars - a presence of $P I I, P I I I$ and $H g I I$ lines in its spectrum (Malaroda, 1981). All theories proposed to explain the presence of heavy elements in the atmospheres of peculiar stars should keep this fact in mind.

A search for elements with $Z>77$ in four cool Ap stars by Faraggiana (1989) using ultraviolet spectra in the 2000-3000 A range gives a negative result in all but one case. Only line of $P b I I \quad \lambda 2203.53$ is present in spectra of two stars HD 188041 and $\gamma E q u$ indicating the overabundance of this element. No trace of $T h I I$ and $U I I$ lines is seen in the spectra of all four Sr-Cr-Eu stars although they belong to the same group as $73 \mathrm{Dra}$, where Severny and Lyubimkov (1986, see also discussion) discovered a 3.0 dex overabundance of these elements. Uranium detection in visible spectral region is based only on $U I I \quad \lambda 3859.58$ line which is strongly blended by $C r I I$ line. The question of actinides presence in Ap stars remains open.

\section{CONCLUSIONS}

1. Average abundances in stars with inhomogeneous surfaces can present approximate relations between abundance anomalies and other characteristics of Ap stars, as well as between anomalies in different groups of stars.

2. There are some elements whose abundances do not strongly depend on magnetic field intensity (for example, Sr-Y-Zr-Ba), but for most of the elements all diffusion calculations should take the magnetic field into account.

3. In some cases ( $, \mathrm{N}, \mathrm{O}, \mathrm{Ga}$, rare-earth elements) abundances obtained from lines in the visual spectral region are an order of magnitude or more higher than those obtained from lines in the infrared or the ultraviolet spectral regions. It is unclear whether this difference is caused by peculiar energy distribution in Ap stars or by non-LTE effects in the upper atmospheres of these stars.

4. Little is known about the connection of surface abundances with the magnetic field 
and the results are sometimes rather inconsistent. To give constraints for theories of the origin of abundance anomalies in Ap stars, it is necessary to obtain self-consistent models of abundance and maagnetic field distribution over the stellar surface. This can be done with the high $S / N$ and high resolution observations in 4 Stokes parameters.

\section{REFERENCES}

Adelman, S.J. 1973, Astrophys. J. 183, 95.

Adelman, S.J. and Cowley, C.R. 1986, in Upper Main Sequence Stars with Anomalous Abundances, eds.C.R. Cowley, M.M. Dworetsky and C. Mégessier D. Reidel Publ. Company, Dordrecht, p.305.

Alecian, G. 1986, in Upper Main Sequence Stars with Anomalous Abundances, eds.C.R. Cowley, M.M. Dworetsky and C. Mégessier, D. Reidel Publ. Company, Dordrecht, p.381.

Alecian, G. and Artru, M.-C. 1987, Astron. Astrophys. 186, 223.

Alecian, G. and Grappin, R. 1984, Astron. Astrophys. 140, 159.

Alecian, G. and Michaud, G. 1981, Astrophys. J. 245, 226.

Allen, M.S. 1977, Astrophys. J. 213, 121.

Allen, M.S. and Cowley, C.R. 1977, Publ. Astron. Soc. Pacif. 89, 386.

Aller, M.F. 1972, Astron. Astrophys. 19, 248.

Artru, M.-C. and Freire-Ferrero, R. 1988, Astron. Astrophys. 203, 111.

Atutov, S.N. and Salagin, A.M. 1988, Pis'ma Astr. Zh. 14, 664.

Bolkal, C., Kocer, D., Duzgelen, A. 1987, Astrophys. Sp. Sci. 139, 295.

Borsenberger, J., Michaud, G., Praderie, F. 1979, Astron. Astrophys. 76, 287.

Borsenberger, J., Michaud, G., Praderie, F. 1981, Astrophys. J. 243, 533.

Borsenberger, J., Michaud, G., Praderie, F. 1984, Astron. Astrophys. 139, 147.

Cowley, C.R. 1976, Astrophys. J. Suppl. 32, 631.

Cowley, C.R. 1980, Vistas in Astronomy 24, 245.

Cowley, C.R. 1983, in Statystical Methods in Astronomy, ed. E.J. Rolfe ESA SP-201, Paris, p.153.

Cowley, C.R. 1984, Phys. Scripta T8, 28.

Cowley, C.R. 1987, Observatory 107, 188.

Cowley, C.R. and Aikman, G.C.L. 1980, Astrophys. J. 242, 684.

Cowley, C.R. and Greenberg, M. 1987, Publ. Astron. Soc. Pacif. 99, 1201.

Faraggiana, R. 1973, Astron. Astrophys. 22, 265.

Faraggiana, R. 1989, Astron. Astrophys. 224, 162.

Faraggiana, R., Gerbaldi, M., Castelli, F., Floquet, M. 1986, Astron. Astrophys. 158, 200.

Floquet, M. 1982, Astron. Astrophys. 112, 299.

Fuhrmann, K. 1989a, Astron. Astrophys. Suppl. Ser. 77, 345.

Fuhrmann, K. 1989b, Astron. Astrophys. Suppl. Ser. 80, 399.

Gel'mukhanov, F.Kh. and Salagin, A.M. 1979, Pis'ma Zh. Eksp. Teor. Fiz. 29, 773.

Gel'mukhanov, F.Kh. and Salagin, A.M. 1980, Zh. Eksp. Teor. Fiz. 78, 1672.

Gerbaldi, M., Faraggiana, R., Molaro, P. 1986, in New Insights in Astrophysics, ESA SP263, Paris, p.49. 
Gerbaldi, M., Floquet, M., Faraggiana, R., van't Veer-Menneret, C. 1989, Astron. Astrophys. Suppl. Ser. 81, 127.

Goncharskij, A.V., Ryabchikova, T.A., Stepanov, V.V., Khokhlova, V.L., Yagola, A.G. 1983, Sov. Astr. 26, 690.

Grevess, N. 1984, Phys. Scripta T8, 49.

Guthrie, B.N.G. 1984, Mon. Not. Roy. Astron. Soc. 206, 85.

Hatzes, A.P. 1988, Ph. D. Dissertation, Univ. of Michigan Microfilms.

Hatzes, A.P. 1990, preprint.

Hatzes, A.P., Penrod, D.G., Vogt, S.S. 1988, Astrophys. J. 341, 456.

Havnes, O. and Conti, P.C. 1971, Astron. Astrophys. 14, 1.

Hric, L. 1988, in Magnetic Stars, eds. I.M. Kopylov and Yu.V. Glagolevskij, Nauka, Leningrad, p.111.

Jacobs, J.M. and Dworetsky, M.M. 1982, Nature 299, 535.

Jaschek, M. and Jaschek, C. 1958, Z. Astrophys. 45, 35.

Khokhlova, V.L. 1983, in Astrophysics and Cosmic Phisics, ed. R.Sunjaev, 24, 233.

Khokhlova, V.L. and Pavlova, V.M. 1984, Pis'ma Astron. Zh. 10, 377.

Krishna Kumar, C., Davila, J.M., Sundar Rajan, R. 1989, Astrophys. J. 337, 414.

Kurucz, R.L. 1979, Astrophys. J. Suppl. 40, 1.

Kurtz, D.W. 1982, Mon. Notic. Roy. Astr. Soc. 200, 807.

Kurtz, D.W. 1988, Mon. Notic. Roy. Astron. Soc. 233, 565.

Kurtz, D.W., Matthews, J.M., Martinez, P., Seeman, J., Cropper, M., Clemens, J.C., Kreidl, T.J., Sterken, C., Schneider, H., Weiss, W.W., Kawaler, S.D., Kepler, S.O., van der Peet, A., Sullivan, D.J. 1989, Mon. Notic. Roy. Astron. Soc. 240, 881.

Landstreet, J.D. 1988, Astrophys. J. 326, 967.

Landstreet, J.D., Barker, P.K., Bohlender, D.A., Jewison, M.S. 1989, Astrophys. J. 344, 876.

Lanz, T. 1985, Astron. Astrophys. 144, 191.

Lanz, T., Artru, M.-C., Didelon, P. 1990, in IAU Symposium 145 Evolution of stars: the photospheric abundance connection, eds. G. Michaud and A. Tutukov, in press.

Lanz, T. and Landstreet, J.D. 1990, in IAU Symposium 145 Evolution of stars: the photospheric abundance connection, eds. G. Michaud and A. Tutukov, in press.

Lebedev, V.S. 1989, Astrophys. Issledov.: Izvestia SAO 27, 54.

Lecrone, D.S. 1976, in Physics of Ap Stars, eds. W.W. Weiss, H. Jenkner and H.J. Wood, Wien Univers., Vienna, p.465.

Lecrone, D.S., Fowler, J.W., Adelman, S.J. 1974, Astron. Astrophys. 32, 237.

Leushin, V.V., Topil'skaya, G.P., Ryabchikova, T.A., Pavlova, V.M. 1990, in IAU Symposium 145 Evolution of stars: the photospheric abundance connection, eds. G. Michaud and A. Tutukov, in press.

Little, S.J. 1974, Astrophys. J. 193, 639.

Loden, L.O. and Sundman A. 1989, J. Astrophys. Astron. 10, 183.

Magazzu, A. and Cowley, C.R. 1986, Astrophys. J. 308, 254.

Malaroda, S. 1981, Publ. Astron. Soc. Pacif. 93, 614.

Mégessier, C. 1984, Astron. Astrophys. 138, 267. 
Mégessier, C. 1986, in Upper Main Sequence Stars with Anomalous Abundances, eds.C.R. Cowley, M.M. Dworetsky and C. Mégessier D. Reidel Publ. Company, Dordrecht, p.275.

Mégessier, C. 1988, Astron. Astrophys. Suppl. Ser. 72, 551.

Mégessier, C., Khokhlova, V.L., Ryabchikova, T.A. 1979, Astron. Astrophys. 71, 295.

Michaud, G. 1970, Astrophys. J. 160, 641.

Michaud, G. 1980, Astron. J. 85, 589.

Michaud, G., Charland, Y., Vauclair, S., Vauclair, G. 1976, Astrophys. J. 210, 447.

Michaud, G, Mégessier, C., Charland, Y. 1981, Astron. Astrophys. 103, 244.

Muthsam, H. 1979, Astron. Astrophys. 73, 159.

Muthsam, H. and Cowley, C.R. 1984, Astron. Astrophys. 130, 348.

North, P. 1984, Astron. Astrophys. 141, 328.

North, P. 1985, Astron. Astrophys. 148, 165.

North, P. and Kroll, R. 1989, Astron. Astrophys. Suppl. Ser. 78, 325.

Osawa, K. 1965, Ann. Tokyo Astron. Obs. 9, ser.2, 123.

Ponomarev, V.O., Smirnov, G.T., Strelnitskij, V.S., Chugai, N.N. 1990, Astron. Tsirk. N 1540,5 .

Proffitt, C.R., Michaud, G. 1989, Astrophys J. 345, 998.

Ramella, M., Gerbaldi, M., Farggiana, R., Bohm, C. 1989, Astron. Astrophys. 209, 233.

Renson, P. 1989, Bull. Var. Stars No. 3452.

Rice, J.B. and Wehlau, W. 1990, Astron. Astrophys. 233, 503.

Roby, S.W. and Lambert, D.L. 1990, Astrophys. J. Suppl. 73, 67.

Ryabchikova, T.A. and Ptitsyn, D.A. 1986, in Upper Main Sequence Stars with Anomalous Abundances, eds. C.R. Cowley, M.M. Dworetsky and C. Mégessier, D. Reidal Publ. Company, Dordrecht, p.319.

Sadakane, K. 1976, Publ. Astron. Soc. Jap. 28, 469.

Sadakane, K., Takada, M., Jugaku, J. 1983, Astrophys. J. 274, 261.

Severny, A.B. and Lyubimkov, L.S. 1986, in Upper Main Sequence Stars with Anomalous Abundances, eds. C.R. Cowley, M.M. Dworetsky and C. Mégessier, D. Reidal Publ. Company, Dordrecht, p.327.

Stępień, K. 1989, Astron. Astrophys. 220, 105.

Steppień, K. and Dominiczak, R. 1989, Astron. Astrophys. 219, 197.

Takada-Hidai, M., Sadakane, K., Jugaku, J. 1986, Astrophys. J. 304, 425.

Totochava, A.G. and Khokhlova, V.L. 1990, Pis'ma Astron. Zh., in press.

Vauclair, S., Vauclair, G., Schatzman, E., Michaud, G. 1978, Astrophys. J. 223, 567.

Vauclair, S. and Vauclair, G. 1982, Ann. Rev. Astron. Astrophys. 20, 37.

Wolff, S.C. 1981, Astrophys. J. 244, 221.

Wolff, S.C. 1983, The A-Stars: Problems and Perspectives, NASA SP-463, Washington, D.C.

Zverko, Y., Hric, L., Zboril, M., Žižňovský, J, Budaj, J. 1990, Bull. Astron. Inst. Czechosl., in press. 